\title{
Extending a perfect matching to a Hamiltonian cycle
}

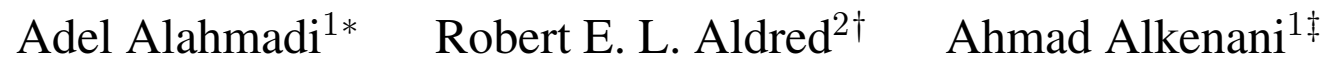

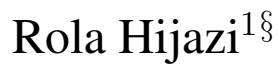 \\ P. Solé ${ }^{1,3} \|^{\top} \quad$ Carsten Thomassen ${ }^{1,4} \mid$ \\ 1 Dept. of Mathematics, Faculty of Science, King Abdulaziz University, Jeddah, Saudi Arabia \\ 2 Dept. of Mathematics and Statistics, University of Otago, Dunedin, New Zealand \\ 3 Telecom ParisTech, France \\ 4 Dept. of Applied Mathematics and Computer Science, Technical University of Denmark, Lyngby, Denmark
}

received $21^{\text {st }}$ May 2014, revised $11^{\text {th }}$ Nov. 2014, $11^{\text {th }}$ Mar. 2015, accepted $12^{\text {th }}$ Mar. 2015.

In 1993 Ruskey and Savage conjectured that in the $d$-dimensional hypercube, every matching $M$ can be extended to a Hamiltonian cycle. Fink verified this for every perfect matching $M$, remarkably even if $M$ contains external edges. We prove that this property also holds for sparse spanning regular subgraphs of the cubes: for every $d \geq 7$ and every $k$, where $7 \leq k \leq d$, the $d$-dimensional hypercube contains a $k$-regular spanning subgraph such that every perfect matching (possibly with external edges) can be extended to a Hamiltonian cycle. We do not know if this result can be extended to $k=4,5,6$. It cannot be extended to $k=3$. Indeed, there are only three 3-regular graphs such that every perfect matching (possibly with external edges) can be extended to a Hamiltonian cycle, namely the complete graph on 4 vertices, the complete bipartite 3-regular graph on 6 vertices and the 3-cube on 8 vertices. Also, we do not know if there are graphs of girth at least 5 with this matching-extendability property.

Keywords: some well classifying words

\section{Introduction}

The $d$-dimensional hypercube $\mathcal{Q}_{d}$ is the $d$-fold Cartesian product of $P_{2}$ s, i.e $P_{2} \square P_{2} \square \ldots \square P_{2}$, with $P_{2}$ appearing $d$ times in the product. The resulting graph is of interest in many disciplines and has been widely studied for many years. It is a Cayley graph of $\mathbb{Z}_{2} \times \mathbb{Z}_{2} \times \ldots \times \mathbb{Z}_{2}$ and is both distance-transitive and bipartite. From the definition it is readily apparent that the graphs are Hamiltonian for $d \geq 2$ and

\footnotetext{
*Email: .

${ }^{\dagger}$ Email: .

†Email: .

§Email: .

IEmail: .

"Email: .
}

1365-8050 @ 2015 Discrete Mathematics and Theoretical Computer Science (DMTCS), Nancy, France 
admit perfect matchings for $d \geq 1$. Indeed, van der Waerden's conjecture (now a theorem, see [3] and [4]) implies a large number of perfect matchings. Kreweras [14] conjectured that every perfect matching of the hypercube extends to a Hamiltonian cycle. This was confirmed in [6] where Fink showed a stronger result, namely that if $K_{\mathcal{Q}_{d}}$ is the complete graph on the same vertex set as $\mathcal{Q}_{d}$ and $M$ is a perfect matching in $K_{\mathcal{Q}_{d}}$, then there is a perfect matching $M^{\prime}$ in $\mathcal{Q}_{d}$ such that $M \cup M^{\prime}$ is a Hamiltonian cycle in $K_{\mathcal{Q}_{d}}$. In [5] the bound on the number of perfect matchings indicated by van der Waerden's conjecture, combined with Fink's proof of the Kreweras conjecture was used to show that there are at least $((d \log 2 /(e \log \log d))(1-$ $o(1)))^{2^{d}}$ Hamiltonian cycles in $\mathcal{Q}_{d}$. In [7] Fink's proof was refined to prove that every perfect matching in $\mathcal{Q}_{d}$ extends to at least $2^{2^{d-4}}$ Hamiltonian cycles.

Kreweras's conjecture is a special case of the following conjecture of Ruskey and Savage [16].

Conjecture 1 Every matching in the hypercube $\mathcal{Q}_{d}$ extends to a Hamiltonian cycle.

Fink's result [6] is a major step toward a solution of Conjecture 1. However, the case of a general matching seems much different from the special case of a perfect matching. Indeed, if one deletes just one edge from the cube, we get a graph which has a matching (namely an odd cut) which does not extend to a cycle. So it is of interest to consider which edges of $\mathcal{Q}_{d}$ may be discarded while maintaining the ability to extend a perfect matching in $K_{\mathcal{Q}_{d}}$ to a Hamiltonian cycle. Gregor [9] showed that, given a perfect matching $M$ in $K_{\mathcal{Q}_{d}}$, we may extend this to a Hamiltonian cycle adding only edges from certain subcubes, depending on $M$. Fon-Der-Flaass [8] showed that we can delete some edges from the cube (at most one edge from each 4-cycle in the cube) and retain the property that every perfect matching extends to a Hamiltonian cycle. In the present paper we show that we may delete most of the edges in the cube and still have a graph in which each perfect matching extends to a Hamiltonian cycle.

Our approach is to extend Fink's result to show that the edges in $\mathcal{Q}_{d}$ that cannot be used to extend a matching $M$ in $K_{\mathcal{Q}_{d}}$ to a Hamiltonian cycle in $M \cup \mathcal{Q}_{d}$ form a matching in $\mathcal{Q}_{d}$. We apply this to prove that, for every $d \geq 7$ and every $k$, where $7 \leq k \leq d$, the $d$-dimensional hypercube contains a $k$-regular spanning subgraph such that every perfect matching (possibly with external edges) can be extended to a Hamiltonian cycle. We do not know if this result can be extended to $k=4,5,6$, see Open Problem 1. It cannot be extended to $k=3$ as we show in Section 3. All of our spanning subgraphs of the cubes have girth 4 . We do not know if there are graphs of girth at least 5 with this matching-extendability property, see Open Problem 4.

Häggkvist [11] studied extendability of perfect matchings in graphs with $p$ vertices ( $p$ even) satisfying the Ore-type condition that the sum of degrees of any two non-adjacent vertices is at least $p+1$. He proved that in such a graph each perfect matching extends to a Hamiltonian cycle. Note that for such graphs it makes no difference if we allow some edges in the perfect matching to be external. Further results and open problems on dense graphs inspired by this result are given in [1], [12], [13].

\section{Preliminaries}

Before proving our main result we introduce some terminology and observations.

Definition: If $A, B$ is a partition of the vertex set of a graph, then the set of edges joining $A, B$ is called a cut. $A, B$ are called the sides of the cut. 
Definition: A perfect matching $M$ in $\mathcal{Q}_{d}$ is called a dimension-cut if $\mathcal{Q}_{d}-M$ is isomorphic to two copies of $\mathcal{Q}_{d-1}$.

We observe that there are precisely $d$ dimension-cuts in $\mathcal{Q}_{d}$ and each edge belongs to a unique dimensioncut. Dimension-cuts are the only matchings in the hypercube that contain cuts. In other words, a matching $M$ separates $\mathcal{Q}_{d}$ (that is, $\mathcal{Q}_{d}-M$ is disconnected) if and only if $M$ is a dimension-cut. The dimensioncuts of $\mathcal{Q}_{d}$ induce a proper $d$-edge-coloring of $\mathcal{Q}_{d}$. With respect to this coloring, a path between a pair of vertices is a shortest path if and only if it contains at most one edge of any color. A given pair of vertices is separated by precisely as many distinct dimension-cuts as there are edges in a shortest path joining them in $\mathcal{Q}_{d}$.

Definition: For any graph $G$ we denote by $K_{G}$ the complete graph on the vertex set $V(G)$.

Definition: A pairing of the graph $G$ is a perfect matching in $K_{G}$.

Definition: For any graph $G$ edges in $E\left(K_{G}\right)-E(G)$ are called external edges.

Clearly, a pairing of $G$ may contain external edges.

Definition: For any graph $G$, we say that a pairing $M$ of $G$ extends to a Hamiltonian cycle if there is a Hamiltonian cycle $C$ of $K_{G}$ in which $E(C)-M \subseteq E(G)$. If every pairing $M$ of $G$ extends to a Hamiltonian cycle, then we say that $G$ has the pairing-Hamiltonian property or, for short, the $\mathrm{PH}$ property.

We may think of the Cartesian product $G \square H$ as a graph obtained from $G$ by replacing every vertex of $G$ by a copy of $H$ and every edge of $G$ by a matching joining two copies of $H$. We call these copies of $H$ in $G \square H$ the canonical copies of $H$ in $G \square H$. Note that there may be other copies of $H$ in $G \square H$ but they will not be called canonical. If $G$ is a path $P_{q}$ with $q$ vertices, then the canonical copies of $H$ in $G \square H$ corresponding to the first and last vertices in $P_{q}$ are called the first and last canonical copies of $H$, respectively.

If $C_{1}, C_{2}$ are two vertex disjoint cycles in a graph $G$, and $G$ contains two edges $e_{1}, e_{2}$ joining two consecutive vertices in $C_{1}$ with two consecutive vertices in $C_{2}$, then we may obtain a cycle $C$ by deleting an edge in each of $C_{1}, C_{2}$ and adding the edges $e_{1}, e_{2}$. We say that $C$ is obtained by merging $C_{1}, C_{2}$.

If $M$ is a matching, and $C$ is a path or cycle, then $C$ is $M$-alternating if every second edge of $C$ is in $M$.

\section{The 3 -regular graphs with the $P H$-property.}

Theorem 1 Let $G$ be a 3-regular graph with the PH-property. Then $G$ is the complete graph $K_{4}$ or the complete bipartite graph $K_{3,3}$ or the 3 -cube $\mathcal{Q}_{3}$.

\section{Proof of Theorem 1:}

It is easy to see that $G$ must be 2-connected. Recall that in a 3-regular graph, vertex-connectivity and edge-connectivity are the same. In the current setting, it is more natural to consider cuts consisting of 
edges.

If $G$ has a cut consisting of two edges $e_{1}=x_{1} x_{2}$ and $e_{2}=y_{1} y_{2}$ joining the sides $A_{1}$ (containing $x_{1}, y_{1}$ ) and $A_{2}$ (containing $x_{2}, y_{2}$ ), then we extend the edges $x_{1} y_{1}, x_{2} y_{2}$ to a pairing of $G$ containing no edge from $A_{1}$ to $A_{2}$. Clearly, that pairing cannot be extended to a Hamiltonian cycle. So $G$ is 3 -connected.

If $G$ has a cut $K$, consisting of a matching with an odd number of edges, each joining side $A_{1}$ to side $A_{2}$, then each $A_{i}$ has an even number of vertices not incident with any edge in $K$. We construct a pairing $M$ of $G$ as follows. Include all edges of $K$ in $M$. Now add edges (possibly external) pairing all vertices in $A_{1}$ not incident with $K$. This is possible since there are evenly many vertices in $A_{1}$ not incident with edges in $K$. Finally, we add edges (again, possibly external) pairing all vertices in $A_{2}$ not incident with $K$ (again there are evenly many such vertices). The only edges in $M$ joining vertices in $A_{1}$ to vertices in $A_{2}$ are those in $K$. Since $|K|$ is odd, $M$ cannot be extended to a Hamiltonian cycle. So we may assume that $G$ has no such cut. In particular, $G$ has girth at least 4 unless $G=K_{4}$.

Consider now a shortest cycle $C: x_{1} x_{2} \ldots x_{k} x_{1}$. Let $y_{i}$ be the neighbor of $x_{i}$ not in $C$, for $i=$ $1,2, \ldots, k$. If $k$ is odd, then the edges leaving $C$ form a matching which is also a cut, a contradiction. So $k$ is even. We may assume that $y_{1}, y_{2}, \ldots, y_{k}$ are distinct since otherwise $k=4$ (by the minimality of $C$ ), in which case $G$ has a cut with three edges forming a matching, unless $G=K_{3,3}$. So consider the matching $x_{1} x_{2}, y_{2} y_{3}, x_{3} x_{4}, \ldots x_{k-1} x_{k}, y_{k} y_{1}$. We extend that matching to a perfect matching and then to a Hamiltonian cycle. It is easy to see that that Hamiltonian cycle must be $x_{1} x_{2} y_{2} y_{3} x_{3} x_{4} \ldots x_{k-1} x_{k} y_{k} y_{1} x_{1}$. In particular $G$ has $2 k$ vertices and girth $k$. It is well known (and easy to see) that a cubic graph with $n \geq 12$ vertices has girth strictly less than $n / 2$. So $G$ has $2 k=8$ vertices. There are only two trianglefree cubic graphs with 8 vertices, namely $\mathcal{Q}_{3}$ and the 8-cycle with 4 diagonals. As those 4 diagonals cannot be extended to a cycle, it follows that $G=\mathcal{Q}_{3}$.

\section{The Cartesian product of a path and a cycle.}

$C_{4} \square K_{4}$ contains $C_{4} \square C_{4}=\mathcal{Q}_{4}$ which has the $P H$-property. Seongmin Ok and Thomas Perrett (private communication) have shown that $C_{q} \square K_{4}$ does not have the $P H$-property when $q \geq 7$, see Section 5 . However, if each matching edge is contained in one of the canonical $K_{4} \mathrm{~s}$, then the matching extends to a Hamiltonian cycle as the proof of the proposition below easily shows. The argument in that proposition will be crucial in our main result.

For this proposition we shall consider the graph $P_{q} \square C_{4}$ to be labelled so that the $q$ canonical 4-cycles are $w_{i} x_{i} y_{i} z_{i} w_{i}$ for $i=1,2, \ldots, q$ and the four canonical paths (when we think of $P_{q} \square C_{4}$ as $C_{4} \square P_{q}$ ) are $w_{1} w_{2} \ldots w_{q}$ etc. Matchings in distinct canonical 4-cycles are said to be similar if they join the same canonical paths. For example, $w_{i} x_{i}, y_{i} z_{i}$ in the $i$-th canonical 4-cycle is similar to the matching $w_{j} x_{j}, y_{j} z_{j}$ in the $j$-th canonical 4-cycle.

Proposition 1 Let $q \geq 3$ be an integer and let $M$ be a perfect matching in $G=P_{q} \square C_{4}$, such that each $M$-edge is contained in a canonical 4-cycle. Assume that not all canonical 4-cycles have similar $M$-matchings. Then $G$ has two M-alternating Hamiltonian paths each starting at $w_{1}$ and ending at diametrically opposite vertices of the last canonical 4-cycle.

\section{Proof of Proposition 1:}

There is a unique $M$-alternating Hamiltonian path starting at $w_{1}$, ending in a vertex in the last canonical 4 -cycle and containing precisely one edge between consecutive canonical 4 -cycles. We shall prove that we 
can find an $M$-alternating Hamiltonian path ending at another vertex of the last canonical 4-cycle (which must be diametrically opposite in the last canonical 4 -cycle because $G$ is bipartite). Consider the case where $M$ has the edges $w_{1} x_{1}, y_{1} z_{1}$ and $w_{2} z_{2}, x_{2} y_{2}$ on first two canonical 4-cycles. The above-mentioned $M$-alternating Hamiltonian path with precisely one edge joining each consecutive pair of canonical 4cycles is then $w_{1} x_{1} y_{1} z_{1} z_{2} w_{2} x_{2} y_{2} y_{3} \ldots$. There is another one, namely $w_{1} x_{1} x_{2} y_{2} y_{1} z_{1} z_{2} w_{2} w_{3} \ldots$. The two Hamiltonian paths enter the third canonical 4-cycle at diametrically opposite points and continue from there in the prescribed manner. Clearly these two Hamiltonian paths must have distinct (diametrically opposite) ends in the last canonical 4-cycle. A similar variation is readily achieved if the first change of matching occurs between canonical 4-cycles $j$ and $j+1$.

\section{The Cartesian product of a complete graph and a cycle.}

We shall seek examples which are the Cartesian product of a complete graph $K_{m}$ and a cycle $C_{q}$. Such a graph is $(m+1)$-regular. If $m$ is odd, we assume that $q$ is even because there should be a pairing.

If $m=2$ then this Cartesian product does not have the extendability property (except if $q=4$ ) because of Theorem 1 .

If $m$ is odd (and hence $q$ is even), then the Cartesian product of $K_{m}$ and $C_{q}$ has an even cut consisting of a matching with $2 m$ edges. If $q \geq 6$, then this matching can be chosen such that the two sides, $A_{1}$ and $A_{2}$, of the cut are odd. (Note, when $q=4$, the only matchings that produce cuts in $K_{m} \square C_{4}$ are those corresponding to perfect matchings in $C_{4}$ and hence both resulting components are even.) But then we extend the cut to a pairing. Since $A_{1}$ and $A_{2}$ are both odd, that pairing contains an odd number of edges between $A_{1}$ and $A_{2}$ and, as such cannot extend to a Hamiltonian cycle.

Theorem 2 The Cartesian product of a complete graph $K_{m}$ (where $m$ is even, $m \geq 6$ ) and a cycle has the PH-property.

We observe that if a graph $G$ has the $P H$-property, then any graph $H$ obtained from $G$ by adding edges also has the $\mathrm{PH}$-property. Consequently, Theorem 2 follows immediately from the following stronger result.

Theorem 3 The Cartesian product of a complete graph $K_{m}$ (where $m$ is even, $m \geq 6$ ) and a path $P_{q}$ with $q \geq 1$ vertices has the PH-property. Moreover, if $M$ is a pairing in which no edge joins vertices from distinct canonical complete graphs, and e is any edge in the last canonical complete graph and not in $M$, then the Hamiltonian cycle containing $M$ can be chosen such that it contains $e$, too.

\section{Proof of Theorem 3:}

Let the graph we consider be denoted $G$.

Clearly, with $q=1, G=K_{m}$ and the result is immediate. So we may assume $q \geq 2$.

Consider first the case where $M$ contains at least one edge which joins two distinct canonical complete graphs $K_{m}$. In this case we adapt Fink's inductive argument from [6] to the current situation as follows. The path $P_{q}$ can be divided into two paths $P_{r}$ and $P_{q-r}$ such that $M$ has edges joining the subgraph of $G$ corresponding to $P_{r}$ with the subgraph corresponding to $P_{q-r}$. Since $m$ is even, there must be an even number of such edges in $M$. Suppose the edges in $M$ between $G_{1}=P_{r} \square K_{m}$ and $G_{2}=P_{q-r} \square K_{m}$ are $e_{1}, e_{2}, \ldots, e_{2 k}$ and that edge $e_{i}=u_{i} v_{i}$ with $u_{i}$ in $V\left(G_{1}\right)$ and $v_{i}$ in $V\left(G_{2}\right)$ for each $i=1,2, \ldots, 2 k$. We form a new pairing $M_{2}$ in $G_{2}$ by adding to those edges of $M$ already joining pairs of vertices in $G_{2}$, the new edges $v_{1} v_{2}, v_{3} v_{4}, \ldots, v_{2 k-1} v_{2 k}$. Now apply induction on $G_{2}$ with $M_{2}$. The edges of $M_{2}$ not in $M$ 
occur in some fixed order on the resulting Hamiltonian cycle in $G_{2}$. Deleting the edges in $M_{2}$ (and not in $M$ ) from the Hamiltonian cycle in $G_{2}$ leaves path segments joining the vertices $v_{i}, i=1,2,3, \ldots, 2 k$ in pairs. We now pair the vertices $u_{1}, u_{2}, \ldots, u_{2 k}$ according to this order and apply induction to $G_{1}$ to yield a Hamiltonian cycle in $G_{1}$ which, when merged with the Hamiltonian cycle in $G_{2}$ gives the desired Hamiltonian cycle in $G$.

Consider next the case where $M$ contains no edge which joins two distinct canonical complete graphs $K_{m}$. Let $e$ be any edge not in $M$ but in the last canonical $K_{m}$. Now we choose another edge $e^{\prime}$ in the last canonical $K_{m}$ such that

(i) $e^{\prime}$ is not in $M$

(ii) $e^{\prime}$ does not join the same two $M$-edges as the two $M$-edges which $e$ joins, and

(iii) the edge $e^{\prime \prime}$ in the second last $K_{m}$ corresponding to $e^{\prime}$ is not in $M$.

As $m \geq 6$ it is easy to find such an edge $e^{\prime}$. Now we let $C$ be a Hamiltonian cycle in the last canonical $K_{m}$ containing both of $e, e^{\prime}$ and also those edges of $M$ which are in that $K_{m}$. We apply induction to $G$ with the last $K_{m}$ removed and with $e^{\prime \prime}$ playing the role of $e$. Then we delete $e^{\prime \prime}$ from the resulting cycle and merge it with $C$ to complete the proof.

It is easy to see that $P_{q} \square K_{4}$ has a perfect matching which cannot be extended to a Hamiltonian cycle when $q \geq 3$. To see this we consider each canonical $K_{4}$ to have vertices $w_{i}, x_{i}, y_{i}, z_{i}, i=1,2, \ldots, q$ and canonical paths $P_{q}$ have vertices $w_{1}, w_{2}, \ldots, w_{q}$ and so on. Taking matchings $w_{1} x_{1}, y_{1} z_{1}$ in the first canonical $K_{4}, w_{2} y_{2}, x_{2} z_{2}$ in the second and $w_{3} z_{3}, x_{3} y_{3}$ in the third we can extend this to a perfect matching in $P_{q} \square K_{4}$ with arbitrarily chosen perfect matchings in each of the remaining $q-3$ canonical $K_{4} \mathrm{~s}$ to yield a perfect matching that cannot extend to a Hamiltonian cycle. Seongmin Ok and Thomas Perrett (private communication) have modified this idea to show that $C_{q} \square K_{4}$ does not have the $\mathrm{PH}$-property for $q \geq 7$.

\section{Extensions of the $\mathrm{PH}$-property for the hypercubes.}

In this section we present a technical extension of Fink's result where we try to extend a matching and an additional prescribed edge to a Hamiltonian cycle.

In the proofs we refer to Fink's inductive argument [6] used to prove his result that any perfect matching $M$ in $K_{\mathcal{Q}_{d}}$ can be extended to a Hamiltonian cycle in $\mathcal{Q}_{d} \cup M$. The argument is as follows. We divide $\mathcal{Q}_{d}$ in to two $(d-1)$-cubes $G_{1}, G_{2}$ joined by a dimension cut. As every pair of vertices in the cube can be divided by some dimension cut, we may assume that $M$ has at least one edge from $G_{1}$ to $G_{2}$. We first apply induction to $G_{1}$. We keep those edges in $M$ which join two vertices in $G_{1}$. We add at random a matching (which we may call new edges) between the ends of those edges in $M$ which go from $G_{1}$ to $G_{2}$. The induction hypothesis gives us a Hamiltonian cycle in $G_{1}$ (plus some external edges). We delete the new edges and get a path system in $G_{1} \cup M$. We transform this path system to new edges in $G_{2}$ and complete the proof by applying the induction hypothesis to $G_{2}$.

The following result will be useful. It is not new having previously appeared in [2, 10, 15], for example. For the sake of completeness, however, we include a short proof.

Proposition 2 (a) If $d \geq 3$ is odd, and $v, v^{\prime}$ are diametrically opposite vertices of $\mathcal{Q}_{d}$, then $\mathcal{Q}_{d}-v-v^{\prime}$ has a Hamiltonian cycle.

(b) If $d \geq 4$ is even, and $v, u$ are neighbors, and $v, v^{\prime}$ are diametrically opposite vertices of $\mathcal{Q}_{d}$, and $u, u^{\prime}$ are diametrically opposite vertices of $\mathcal{Q}_{d}$, then $\mathcal{Q}_{d}-v-v^{\prime}-u-u^{\prime}$ has a Hamiltonian cycle. 
Proof of Proposition 2: We first prove (a). If $d=3$, then $\mathcal{Q}_{d}-v-v^{\prime}$ is a cycle of length 6 . So assume that $d \geq 5$. We think of the $d$-cube $\mathcal{Q}_{d}$ as the product $\mathcal{Q}_{d-2} \square \mathcal{Q}_{2}$. In other words, $\mathcal{Q}_{d}$ is obtained from $\mathcal{Q}_{d-2}$ by replacing every vertex by a canonical 4 -cycle. Consider a Hamiltonian cycle $C$ in $\mathcal{Q}_{d-2}$ and suppose $C$ has some fixed direction imposed upon it. We transform $C$ into a cycle in $\mathcal{Q}_{d}-v-v^{\prime}$ as follows. In the canonical 4-cycle containing $v$, we build a path $x y z$ of length 2 containing the three other vertices in this canonical 4-cycle. In the $\mathcal{Q}_{d-2}$ containing $z$, we proceed from $z$ to a neighboring canonical 4-cycle using the directed edge in $C$. In that canonical 4-cycle we pick up all four vertices (note that we can do this in two distinct ways) and proceed to a neighboring canonical 4-cycle using the directed edge in $C$ in our current canonical $\mathcal{Q}_{d-2}$. We proceed like this except when we reach the canonical 4-cycle containing $v^{\prime}$. We hit this 4-cycle at a neighbor of $v^{\prime}$ because $d$ is odd and $\mathcal{Q}_{d}$ is bipartite. Then we pick up the three vertices distinct from $v^{\prime}$ (this can be done in exactly one way) and continue. When we return to the first canonical 4-cycle, we hit that canonical 4-cycle either at $x$ or $z$ because $\mathcal{Q}_{d}$ is bipartite and $v, v^{\prime}$ belong to distinct partite classes. If we hit at $x$, we are done. If we hit at $z$, we can easily modify our path in some canonical 4-cycle traversing the vertices in the opposite direction around the canonical 4-cycle. In this way we terminate at $x$ and get a cycle containing all vertices, except $v, v^{\prime}$.

We now prove (b). We think of the $d$-cube $\mathcal{Q}_{d}$ as the union of two $(d-1)$-cubes $G_{1}, G_{2}$ joined by the dimension cut containing the edge $v u$. Then $v, u^{\prime}$ are diametrically opposite in $G_{1}$, and $u, v^{\prime}$ are diametrically opposite in $G_{2}$. By (a), $G_{1}-v-u^{\prime}$ has a Hamiltonian cycle $C_{1}$. Let $C_{2}$ be the corresponding Hamiltonian cycle in $G_{2}-u-v^{\prime}$. Then we complete the proof of (b) by merging $C_{1}, C_{2}$.

Again, we think of the $d$-cube $\mathcal{Q}_{d}$ as the product $\mathcal{Q}_{d-2} \square \mathcal{Q}_{2}$. In other words, $\mathcal{Q}_{d}$ is obtained from $\mathcal{Q}_{d-2}$ by replacing every vertex by a canonical 4 -cycle.

Proposition 3 Let $d \geq 3$ be an integer and let $M$ be a pairing in $\mathcal{Q}_{d}=\mathcal{Q}_{d-2} \square \mathcal{Q}_{2}$, such that every edge in $M$ joins two vertices in the same canonical 4-cycle. Let e be any edge in $\mathcal{Q}_{d}$ joining two distinct canonical 4-cycles. Then $\mathcal{Q}_{d}$ has a perfect matching $M^{\prime}$ such that $M^{\prime}$ contains e and $M \cup M^{\prime}$ is a Hamiltonian cycle in $\mathcal{Q}_{d} \cup M$.

Proof of Proposition 3: The proof is by induction on $d$. Suppose first that $d=3$. Then there are two canonical 4-cycles, and $e$ is an edge joining them. If the two matchings in the two canonical 4-cycles are non-similar, then the union of $M$ and the four edges joining the two canonical 4-cycles form a Hamiltonian cycle. That Hamiltonian cycle contains $e$. If the two matchings in the two canonical 4-cycles are similar, then there is a Hamiltonian cycle containing $M$ and two edges joining the two canonical 4-cycles and also another Hamiltonian cycle containing $M$ and two other edges joining the two canonical 4-cycles. One of these two Hamiltonian cycles contains $e$.

Assume now that $d \geq 4$. We refine our view of $\mathcal{Q}_{d}$ further noting that each $\mathcal{Q}_{d-2}=\mathcal{Q}_{1} \square \mathcal{Q}_{d-3}$ (i.e. two copies of $\mathcal{Q}_{d-3}$ joined by a dimension cut). Furthermore, we may assume that $e$ lies inside one of the canonical $\mathcal{Q}_{d-3} \mathrm{~s}$ in this product. This decomposition imposes a natural perspective on $\mathcal{Q}_{d}$ as the union of two $(d-1)$-cubes $G_{1}, G_{2}$ joined by a dimension cut not containing $e$. Moreover, each $G_{i}$ inherits its structure as $\mathcal{Q}_{d-3} \square \mathcal{Q}_{2}$ from our original cube. We may assume that $e$ is in $G_{1}$ and apply induction to $G_{1}$. The resulting cycle which contains $e$ contains another edge $e_{1}$ in the same dimension cut as $e$. Let $e_{2}$ be the edge in $G_{2}$ corresponding to $e_{1}$. Now apply induction to $G_{2}$ with $e_{2}$ playing the role of $e$. Then merge the two cycles by deleting $e_{1}, e_{2}$ and adding two edges between $G_{1}, G_{2}$. 
Theorem 4 Let $d \geq 2$ be an integer and let $M$ be a pairing in $\mathcal{Q}_{d}$. Let $e_{1}, e_{2}$ be two edges in $\mathcal{Q}_{d}-M$ incident with the same vertex. Then $\mathcal{Q}_{d}$ has a perfect matching $M^{\prime}$ such that $M^{\prime}$ contains one of $e_{1}, e_{2}$ and $M \cup M^{\prime}$ is a Hamiltonian cycle in $\mathcal{Q}_{d} \cup M$.

Proof of Theorem 4: The proof is by induction on $d$. For $d=2$, the statement is trivial. So assume that $d \geq 3$.

There is a unique 4 -cycle $C_{1}$ in $\mathcal{Q}_{d}$ containing $e_{1}, e_{2}$. Now we think of $\mathcal{Q}_{d}$ as $\mathcal{Q}_{d-2} \square \mathcal{Q}_{2}$ such that $e_{1}, e_{2}$ are contained in a common canonical 4-cycle $C_{1}$. Moreover, we shall label the vertices in $C_{1}$, $w_{1}, x_{1}, y_{1}, z_{1}$ where $e_{1}=w_{1} x_{1}$ and $e_{2}=w_{1} z_{1}$. We consider the remaining canonical 4-cycles with respect to this decomposition to be labelled $C_{2}, C_{3}, \ldots, C_{2^{d-2}}$ and their constituent vertices to be labelled analogously to those in $C_{1}$.

We distinguish two cases:

Case 1: Each edge in $M$ joins two vertices in the same canonical 4-cycle;

Case 2: There is at least one edge $e=u v$ in $M$ such that $u \in V\left(C_{i}\right)$ and $v \in V\left(C_{j}\right)$ with $i \neq j$.

\section{Proof of Case 1:}

Note, since neither $e_{1}$ nor $e_{2}$ is in $M$, the vertices in $C_{1}$ are paired by diagonal edges external to $\mathcal{Q}_{d}$. If $d=3$, it is easy to find $M^{\prime}$. So assume that $d \geq 4$. We think of $\mathcal{Q}_{d-2}$ as two $(d-3)$-cubes $G_{1}^{\prime}, G_{2}^{\prime}$ joined by a dimension cut. So, we may think of $\mathcal{Q}_{d}$ as the union of $G_{1}, G_{2}$ joined by a dimension cut, where each of $G_{1}, G_{2}$ is the Cartesian product of a $(d-3)$-cube with $\mathcal{Q}_{2}$. We may assume that $G_{1}$ contains $e_{1}, e_{2}$ and apply induction to $G_{1}$. The resulting Hamiltonian cycle contains an edge $e^{\prime}$ joining two vertices in distinct canonical 4-cycles. Let $e$ be the corresponding edge in $G_{2}$. Now apply Proposition 3 to $G_{2}$. Then we merge the two cycles after deleting the edges $e, e^{\prime}$. This completes the proof of Case 1 .

\section{Proof of Case 2:}

We now assume that some edge of $M$ joins two vertices in distinct canonical 4-cycles.

If $d=3$, then $M$ contains either two or four edges joining the two canonical 4-cycles. Fink's proof [6] shows that $\mathcal{Q}_{3} \cup M$ has a Hamiltonian cycle whose edges between the canonical 4-cycles are precisely those edges in $M$ which join these canonical 4-cycles. Such a Hamiltonian cycle must contain at least one of $e_{1}, e_{2}$. So assume that $d \geq 4$.

Again, we think of $\mathcal{Q}_{d-2}$ as two $(d-3)$-cubes joined by a dimension cut. This induces a decomposition of $\mathcal{Q}_{d}$ into $G_{1}, G_{2}$ joined by a dimension cut. Note that in this decomposition, each $G_{i}$ is a $\mathcal{Q}_{d-3} \square \mathcal{Q}_{2}$ in which the canonical $\mathcal{Q}_{2}$ s are canonical 4-cycles denoted $C_{1}, C_{2}, \ldots C_{2^{d-2}}$. We shall refer to such decompositions as very canonical decompostions of $\mathcal{Q}_{d}$. As before, we assume that $G_{1}$ contains $C_{1}$ and hence $e_{1}, e_{2}$ but now we may also assume that some edge in $M$ joins $G_{1}, G_{2}$.

We look to apply the inductive hypothesis to $G_{1}$ and then Fink's result to $G_{2}$. To apply the inductive argument to $G_{1}$, we form a pairing of $G_{1}$ as follows. Keep those edges in $M$ which join two vertices in $G_{1}$. For those edges in $M$ which join vertices in $G_{1}$ with vertices in $G_{2}$ we arbitrarily choose a matching that pairs up the (even number) of endvertices of edges in $M$ whose other ends lie in $G_{2}$. We shall refer to this pairing in $G_{1}$ as $M_{1}$.

We distinguish two further subcases.

Case 2.1: For some very canonical decomposition of $\mathcal{Q}_{d}$ into $G_{1}, G_{2}$, with $M$ containing edges joining $G_{1}$ to $G_{2}$, the pairing $M_{1}$ can be chosen so that $\left\{e_{1}, e_{2}\right\} \cap M_{1}=\emptyset$.

\section{Proof of Case 2.1:}


In this case, we choose $M_{1}$ containing neither of $e_{1}, e_{2}$ and we complete the proof by applying induction to $G_{1}$ and then Fink's argument to $G_{2}$.

Case 2.2: For each very canonical decomposition of $\mathcal{Q}_{d}$ into $G_{1}, G_{2}$, with edges in $M$ joining $G_{1}$ to $G_{2}$, the pairing $M_{1}$ is forced to contain either $e_{1}$ or $e_{2}$.

\section{Proof of Case 2.2:}

Clearly, if we have a canonical decomposition of $\mathcal{Q}_{d}$ in which more than two edges of $M$ join vertices in $G_{1}$ to vertices in $G_{2}$, we can choose $M_{1}$ to avoid $e_{1}$ and $e_{2}$.

We note that for each pair of canonical 4-cycles, $C_{i}, C_{j}, i \neq j$, there is a canonical decomposition of $\mathcal{Q}_{d}$ that separates $C_{i}$ from $C_{j}$. Thus, if $M$ contains an edge $f=t t^{\prime}$ with $t \in V\left(C_{i}\right)$ and $t^{\prime} \in V\left(C_{j}\right)$, $i \neq j$ such that $\left\{t, t^{\prime}\right\} \cap\left\{w_{1}, x_{1}, z_{1}\right\}=\emptyset$, then we can find a canonical decomposition of $\mathcal{Q}_{d}$ that puts us in Case 2.1. Consequently, we may assume that $M$ contains precisely two edges joining vertices in different canonical 4-cycles. Moreover, one of these edges is incident with $w_{1}$ while the other is incident with either $x_{1}$ or $z_{1}$. As such, we may assume, without loss of generality that the only two edges in $M$ joining canonical 4-cycles are $f_{1}=w_{1} t$ and $f_{2}=x_{1} t^{\prime}$. Note, by parity, both $t$ an $t^{\prime}$ are vertices in the same canonical 4-cycle, $C_{2}$, say.

Case 2.2.1: The very canonical decomposition of $\mathcal{Q}_{d}$ into $G_{1}, G_{2}$ can be chosen such that there are no edges in $M$ between $G_{1}, G_{2}$.

\section{Proof of Case 2.2.1:}

We note that in $G_{2}$ no edge of $M$ can join a pair of vertices in different canonical 4-cycles. Thus we apply induction to $G_{1}$, and then apply Proposition 3 to $G_{2}$ where $e$ is an appropriate edge which can be used to merge the two cycles.

Case 2.2.2: For each very canonical decomposition of $\mathcal{Q}_{d}$ into $G_{1}, G_{2}$, (where $C_{1}$ is in $G_{1}$ ), there are precisely two $M$-edges between $G_{1}, G_{2}$, and these two $M$-edges are $f_{1}=w_{1} t$ and $f_{2}=x_{1} t^{\prime}$, join the two ends of $e_{1}=w_{1} x_{1}$ to two vertices in a single canonical 4-cycle, $C_{2}$ (i.e. $\left\{t, t^{\prime}\right\} \subset\left\{w_{2}, x_{2}, y_{2}, z_{2}\right\}$ ).

\section{Proof of Case 2.2.2:}

Since this applies to all partitions of $\mathcal{Q}_{d}$, the two canonical 4-cycles $C_{1}, C_{2}$ correspond to diametrically opposite vertices $v, v^{\prime}$ in $\mathcal{Q}_{d-2}$.

As usual, we think of $\mathcal{Q}_{d}$ as being obtained from $\mathcal{Q}_{d-2}$ by replacing every vertex by a canonical 4cycle. Let $C_{0}$ be a fixed Hamiltonian cycle in $\mathcal{Q}_{d-2}$. We use $C_{0}$ to construct a Hamiltonian cycle in $\mathcal{Q}_{d} \cup M$ containing $M$. First construct a path starting with the vertex $z_{1}$ and the edge $z_{1} y_{1}$ (which is in $M$ ) followed by the edge corresponding to an edge in $C_{0}$. After that we follow $C_{0}$ and pick up the two $M$-edges in the canonical 4-cycle corresponding to each vertex of $C_{0}$. When we encounter $C_{2}$, the canonical 4-cycle corresponding to $v^{\prime}$, we pick up the $M$-edge joining two vertices in $C_{2}$, and we also include the path $t w_{1} x_{1} t^{\prime}$. In similar fashion, we follow the rest of $C_{0}$, picking up each pair of edges from $M$ in each canonical 4-cycle along the way. If this path terminates at $z_{1}$ we have finished. So assume that it terminates at $x_{1}$. (It cannot terminate at $w_{1}$ or $y_{1}$ because the cube is bipartite.) Since it must terminate at $x_{1}$, it follows that we have no choice in this procedure. This implies that all $M$-edges are internal except the two edges $w_{1} t, x_{1} t^{\prime}$ and that $t, t^{\prime}$ must be consecutive on $C_{2}$.

In each canonical 4-cycle there are two perfect matchings, namely the ones contained in the dimension cut containing $e_{1}$, which we call horizontal edges and the ones contained in the dimension cut containing $e_{2}$ which we call vertical edges. We focus again on the Hamiltonian cycle $C_{0}$ in $\mathcal{Q}_{d-2}$. The proof of 
Proposition 1 shows that we may assume that all matchings in the canonical 4-cycles are horizontal. Also, the edge in $M$ joining two vertices in $C_{2}$ is horizontal. Otherwise the proof of Proposition 1 would give us a choice we could use to get the desired Hamiltonian cycle. We now consider the $M$-alternating cycle $S$ of length 8 containing all vertices in the 4 -cycles $C_{1}, C_{2}$. If $d=4$ we first use the two non- $M$ edges in $E\left(C_{2}\right) \cap E(S)$ to merge the two remaining 4-cycles, $C_{3}, C_{4}$, with the cycle $S$ to form the desired Hamiltonian cycle. If $d \geq 5$ and $d$ is odd, then, using Proposition 2 (a), we consider a Hamiltonian cycle in $\mathcal{Q}_{d-2}-v-v^{\prime}$ to find an $M$-alternating cycle $S^{\prime}$ containing all $M$ edges not in $S$. Then we merge $S, S^{\prime}$ using a vertical edge in each of $S, S^{\prime}$. If $d \geq 6$ and $d$ is even, then we argue in the same way, except that $\mathcal{Q}_{d-2}-v-v^{\prime}$ has no Hamiltonian cycle because $v, v^{\prime}$ are in the same bipartite class. Instead we consider, using Proposition 2 (b), a Hamiltonian cycle in $\mathcal{Q}_{d-2}-v-v^{\prime}-u-u^{\prime}$ where also $u, u^{\prime}$ are diametrically opposite, and $u, u^{\prime}$ are neighbors to $v, v^{\prime}$, respectively, to find an $M$-alternating cycle $S^{\prime \prime}$, containing all $M$-edges which are neither in $S$ nor the two canonical 4-cycles corresponding to $u, u^{\prime}$. Then we first merge $S^{\prime \prime}, S$ using a vertical edge in each of $S^{\prime \prime}, S$, and then we merge the resulting cycle with each of the canonical 4 -cycles corresponding to $u, u^{\prime}$, respectively.

Theorem 4 shows that those edges which cannot be extended together with $M$ to a Hamiltonian cycle form a matching. We do not know how big this matching can be, see Open Problem 2 below. But the following example shows that it may have two edges. Consider a dimension cut dividing the cube into two subcubes $G_{1}, G_{2}$. Let $e_{1}=x_{1} x_{2}$ and $e_{2}=y_{1} y_{2}$ be two of the edges in the dimension cut. In the dimension cut we now replace $e_{1}, e_{2}$ by the two external edges $x_{1} y_{2}, x_{2} y_{1}$. The resulting perfect matching (pairing as it contains two external edges) can be extended to a Hamiltonian cycle, but such a Hamiltonian cycle cannot contain either of $e_{1}, e_{2}$.

\section{Sparse regular spanning subgraphs of the hypercubes with the $P H$-property.}

In this section we use the previous results to prove the main result that it is possible to remove most of the edges of the hypercube and preserve the remarkable $\mathrm{PH}$-property. It follows that the same holds for the graphs in Theorems 2 and 3 when $m$ is a power of 2 .

Theorem 5 Let $d \geq 5$ be an integer and let $M$ be a pairing in $P_{q} \square \mathcal{Q}_{d}$. Then $M$ can be extended to a Hamiltonian cycle in $M \cup P_{q} \square \mathcal{Q}_{d}$. Moreover, if each edge of $M$ joins two vertices in the same canonical $\mathcal{Q}_{d}$, then the last $\mathcal{Q}_{d}$ contains a matching $M^{\prime}$ such that, for each edge e in the last $\mathcal{Q}_{d}$, but not in $M^{\prime}, M$ can be extended to a Hamiltonian cycle of $M \cup P_{q} \square \mathcal{Q}_{d}$ containing $e$.

Proof of Theorem 5: The proof is by induction on $q$. For $q=1,2$, Theorem 5 follows from Theorem 4 because $P_{1} \square \mathcal{Q}_{d}$ and $P_{2} \square \mathcal{Q}_{d}$ are cubes. So assume that $q \geq 3$.

If some $M$-edge joins two distinct canonical $\mathcal{Q}_{d}$ s, then we apply Fink's inductive argument. So assume that each $M$-edge joins two vertices in the same canonical $\mathcal{Q}_{d}$.

Now apply induction to $P_{q-1} \square \mathcal{Q}_{d}$ which we think of as $P_{q} \square \mathcal{Q}_{d}$ with the last canonical $\mathcal{Q}_{d}$ deleted. The induction hypothesis results in a matching $M^{\prime}$ in the last canonical $\mathcal{Q}_{d}$ in $P_{q-1} \square \mathcal{Q}_{d}$.

Suppose now (reductio ad absurdum) that Theorem 5 is false. Then the last canonical $\mathcal{Q}_{d}$ in $P_{q} \square \mathcal{Q}_{d}$ contains two edges $e_{1}, e_{2}$ incident with the same vertex $w_{1}$ such that $M$ cannot be extended to a Hamiltonian cycle using edges in $P_{q} \square \mathcal{Q}_{d}$, one of which is $e_{1}$ or $e_{2}$. (Clearly, we may assume that $e_{1}, e_{2}$ are not in $M$.) We shall show that this leads to a contradiction. 
For this we consider a dimension cut in the last $\mathcal{Q}_{d}$ dividing it into two $(d-1)$-cubes, $G_{1}, G_{2}$, such that $M$ has, say, $r$ edges joining $G_{1}, G_{2}$, where $r \geq 2$. Assume that $w_{1}$ is in $G_{2}$.

Case 1: The dimension cut can be chosen such that $e_{1}, e_{2}$ belong to $G_{2}$.

Now we try to apply Theorem 4 to $G_{2}$ after the $r$ edges in $M$ joining vertices in $G_{1}$ to vertices in $G_{2}$ have been replaced by $r / 2$ new (possibly external) edges in $G_{2}$.

Subcase 1.1: The $r / 2$ new (possibly external) edges in $G_{2}$ can be chosen such that none of them is $e_{1}$ or $e_{2}$.

In this subcase we apply Theorem 4 to $G_{2}$ such that the resulting Hamiltonian cycle $C$ contains one of $e_{1}, e_{2}$. Deleting the new edges in $G_{2}$, we obtain a path system in $G_{2}$. As in Fink's inductive argument, that path system gives rise to a pairing in $G_{1}$, maintaining all edges of $M$ contained in $G_{1}$. We apply Theorem 4 to that pairing. We select any vertex $x$ in $G_{1}$ and we select two edges $e_{1}^{\prime}, e_{2}^{\prime}$ in $G_{1}$ and incident with $x$ such that neither of $e_{1}^{\prime}, e_{2}^{\prime}$ is a matching edge (in the new matching in $G_{1}$ ), and neither of $e_{1}^{\prime}, e_{2}^{\prime}$ correspond to an edge in $M^{\prime}$. Since $G_{1}$ is a $(d-1)$-cube, this cube has $d-1$ edges incident with $x$. Thus $e_{1}^{\prime}, e_{2}^{\prime}$ can be chosen among $d-3$ edges incident with $x$. As $d \geq 5$, this is possible. So, we can apply Theorem 4 to $G_{1}$. Without loss of generality, we may assume that the resulting Hamiltonian cycle $C^{\prime}$ contains $e_{1}^{\prime}$, say. Now we apply the induction hypothesis to $P_{q-1} \square \mathcal{Q}_{d}$ in order to get a Hamiltonian cycle $C^{\prime \prime}$ containing the $M$-edges in that graph and also containing the edge corresponding to $e_{1}^{\prime}$ (as that edge is not in $M^{\prime}$ ). Now we can first combine $C, C^{\prime}$ into an $M$-alternating cycle $C^{\prime \prime \prime}$ containing all vertices in the last canonical $\mathcal{Q}_{d}$ and the edge $e_{1}^{\prime}$. Finally, we merge $C^{\prime \prime \prime}$ with $C^{\prime \prime}$ (after deleting $e_{1}^{\prime}$ and the corresponding edge in $C^{\prime \prime}$ ) to get the desired Hamiltonian cycle in $M \cup P_{q} \square \mathcal{Q}_{d}$. This contradiction completes Subcase 1.1.

Subcase 1.2: The $r / 2$ new (possibly external) edges in $G_{2}$ cannot be chosen such that none of them is $e_{1}$ or $e_{2}$.

In this case must have $r=2$, and the two $M$-edges from $G_{2}$ to $G_{1}$ are incident with $w_{1}$ and an end $x_{1}$ of $e_{1}$ or $e_{2}$, say $e_{1}$. Again, we think of the last canonical $d$-cube $\mathcal{Q}_{d}$ in $P_{q} \square \mathcal{Q}_{d}$ as the product $\mathcal{Q}_{d-2} \square \mathcal{Q}_{2}$. In other words, $\mathcal{Q}_{d}$ is obtained from $\mathcal{Q}_{d-2}$ by replacing each vertex by a canonical 4 -cycle. We may assume that one of these canonical 4-cycles is $C_{1}: w_{1} x_{1} y_{1} z_{1} w_{1}$ where $e_{1}=w_{1} x_{1}, e_{2}=w_{1} z_{1}$. The assumption of Subcase 1.2 implies that the two $M$-edges incident with $w_{1}, x_{1}$ join $C_{1}$ to another canonical 4-cycle $C_{i}$ and all other $M$-edges join two vertices in the same canonical 4-cycle. We now consider a Hamiltonian cycle of $\mathcal{Q}_{d-2}$. We let $C_{1}, C_{2}, \ldots, C_{2^{d-2}}, C_{1}$ be the corresponding cyclic sequence of canonical 4-cycles in the last canonical $d$-cube $\mathcal{Q}_{d}$ in $P_{q} \square \mathcal{Q}_{d}$. We transform that to a Hamiltonian cycle in last canonical $d$-cube $\mathcal{Q}_{d}$ with its $M$-edges added such that the Hamiltonian cycle contains all those $M$-edges. We begin with the edge from $y_{1}$ to $C_{2}$. We pick up the two $M$-edges joining vertices in $C_{2}$ and proceed to $C_{3}$. When we reach $C_{i}$ we also pick up $w_{1}, x_{1}$.

Suppose that we have a choice in some $C_{j}$. Then we can make sure that we hit $z_{1}$ when we terminate in $C_{1}$ so that we obtain a Hamiltonian cycle. As any of the $d-2$ edges from $y_{1}$ to another canonical 4 -cycle can play the role of the first edge in this Hamiltonian cycle, we can choose the first edge $e^{\prime}$ such that it does not correspond to an edge in $M^{\prime}$ or an edge in $M$ in the last canonical $d$-cube $\mathcal{Q}_{d}$ in $P_{q-1} \square \mathcal{Q}_{d}$. So, we can extend the $M$-edges in $P_{q-1} \square \mathcal{Q}_{d}$ to a Hamiltonian cycle containing the edge $e^{\prime \prime}$ corresponding to $e^{\prime}$. By deleting the edges $e^{\prime}, e^{\prime \prime}$ we now merge the two Hamiltonian cycles into one which contains $e_{1}$ and gives a contradiction. So we may assume that we have no choice when we transform the sequence $C_{1}, C_{2}, \ldots$ into a Hamiltonian cycle. 
Since we have no choice, we conclude that both $M$-edges joining vertices in $C_{j}$ are also edges in this $C_{j}$, except that precisely one $M$-edge is an edge in $C_{i}$. Moreover, we may assume that all these matchings the cycles $C_{j}$ are similar, since otherwise, we modify the cycle in two consecutive $C_{j}, C_{j+1}$ to obtain the desired choice. So, we may assume that all $M$-edges in the last canonical $d$-cube, except the two incident with $w_{1}, x_{1}$, are contained in the dimension cut containing $e_{1}=w_{1} x_{1}$. We may also assume that the two $M$-edges incident with the ends of $e_{1}$ join $w_{1}, x_{1}$ with either $w_{i}, x_{i}$ or $x_{i}, w_{i}$ or $y_{i}, z_{i}$ or $z_{i}, y_{i}$, respectively.

We now focus on the sequence $C_{1}, C_{2}, \ldots, C_{2^{d-2}}$ of canonical 4-cycles in the last canonical $d$-cube $\mathcal{Q}_{d}$ in $P_{q} \square \mathcal{Q}_{d}$ (rather than the cyclic sequence $C_{1}, C_{2}, \ldots, C_{2^{d-2}}, C_{1}$ ). Let $P_{1}$ be the path $w_{1} z_{1} y_{1} y_{2} z_{2} z_{3}$ $\ldots x_{2} x_{1}$ such that $P_{1}$ contains all vertices in $C_{1} \cup C_{2} \cup \ldots \cup C_{i-1}$ and all $M$-edges in these cycles. So $P_{1}$ zig-zags through the $y, z$ vertices in $C_{1}, \ldots C_{i-1}$ and zig-zags back through the $w, x$ vertices in $C_{i-1} \ldots C_{1}$. The exact transition at $C_{i-1}$ depends on whether $i$ is odd or even. We let $P_{2}$ denote the path $w_{i} w_{i+1} x_{i+1} x_{i+2} \ldots y_{i+1} z_{i+1} z_{i}$ such that $P_{2}$ starts and terminates at $C_{i}$ and contains all vertices of $C_{i+1} \cup C_{i+2} \cup \ldots \cup C_{2^{d-2}}$ and all $M$-edges in these cycles. Again, $P_{2}$ zig-zags through the $w, x$ vertices in $C_{i}, \ldots C_{2^{d-2}}$ and back through the $y, z$ vertices. The exact transition again being dependent on whether $i$ is odd or even. Now $P_{1} \cup P_{2}$ can be extended to the desired Hamiltonian cycle containing all edges of $M$. As it also contains the edge $e_{2}$ we have reached a contradiction. The exact details of how the paths $P_{1}$ and $P_{2}$ are combined depends on the $M$ edges between $\left\{w_{1}, x_{1}\right\}$ and either $\left\{w_{i}, x_{i}\right\}$ or $\left\{y_{i}, z_{i}\right\}$. The variations are quite straightforward and are left to the reader.

Case 2: The dimension cut cannot be chosen such that $e_{1}, e_{2}$ belong to $G_{2}$.

In this case all $M$-edges in the last canonical $d$-cube join two vertices in the same canonical 4 -cycle $C_{j}$. This case is similar to Subcase 1.2, except that it is easier since we need not worry about the $C_{i}$ which appears in Subcase 1.2.

As $\mathcal{Q}_{r+d}$ has a spanning subgraph which is isomorphic to $C_{2^{r}} \square \mathcal{Q}_{d}$ which is $(d+2)$-regular and has a spanning subgraph isomorphic to $P_{2^{r}} \square \mathcal{Q}_{d}$, it follows that the graphs in Theorem 4 can also be realized by spanning subgraphs of cubes for each $k \geq 7$.

We do not know if $C_{q} \square \mathcal{Q}_{d}$ has the $P H$-property for $d=3,4$, see Open Problem 1 below.

\section{Open problems}

In this section we summarize the open problems mentioned earlier.

Open Problem 1 Does $C_{q} \square \mathcal{Q}_{d}$ have the PH-property for $d=3,4$ ?

Theorem 4 shows that those edges which cannot be extended together with a perfect matching $M$ to a Hamiltonian cycle form a matching. As mentioned earlier, we do not know how big this matching can be. Specifically, we suggest the following problem.

Open Problem 2 Let $d \geq 2$ be an integer and let $M$ be a pairing in $\mathcal{Q}_{d}$. Does $\mathcal{Q}_{d}$ contain a matching $M^{\prime}$ with at most 100 edges such that, for every edge e in $\mathcal{Q}_{d}-M^{\prime}, \mathcal{Q}_{d}$ contains a perfect matching $M^{\prime \prime}$ containing e such that $M \cup M^{\prime \prime}$ is a Hamiltonian cycle?

In this paper we have focused primarily on sparse graphs with the $P H$-property which are subgraphs of the cubes. It may also be of interest to study the $P H$-property for general sparse graphs. In particular, it would be interesting to find a counterpart of Theorem 3 for 4-regular graphs. We suggest the following: 
Open Problem 3 For which values of $p, q$ does $C_{p} \square C_{q}$ have the PH-property?

We showed in Section 5 that $P_{q} \square K_{4}$ has a perfect matching that cannot be extended to a perfect matching when $q \geq 3$. Hence $P_{q} \square C_{4}$ does not have the $P H$-property for $q \geq 3$. The same argument shows that $C_{q} \square C_{4}$ does not have the $P H$-property except for finitely many $q$. Seongmin Ok and Thomas Perrett (private communication) have shown, by a similar argument, that even $C_{q} \square K_{4}$ does not have the $P H$-property when $q \geq 7$.

The square of a cycle $C_{p}^{2}$ is obtained from a cycle $C_{p}$ by adding all edges joining vertices of distance 2 on the cycle. Seongmin Ok and Thomas Perrett (private communication) have also shown that $C_{p}^{2}$ does not have the $P H$-property when $p$ is large.

On the positive side, Seongmin Ok and Thomas Perrett (private communication) have informed us that they have modified the method of Theorem 3 to obtain an infinite class of 4-regular graphs with the $\mathrm{PH}$ property, namely each graph which can be obtained from a cycle $C_{p}, p \geq 3$ by replacing each vertex by two vertices and replacing each edge by the four edges joining the corresponding pairs of vertices.

We do not know of graphs of girth at least 5 and with the $P H$-property.

Open Problem 4 Are there infinitely many graphs of girth at least 5 and with the PH-property?

\section{Acknowledgements}

This paper was funded by the Deanship of Scientific Research (DSR), King Abdulaziz University, under grant No. (33-3/1432/HiCi). The authors, therefore, acknowledge with thanks DSR technical and financial support.

\section{References}

[1] K.A. Berman, Proof of a conjecture of Häggkvist on cycles and independent edges, Discrete Mathematics, 46 (1983) 9-13.

[2] T. Dvořák, P. Gregor, Partitions of faulty hypercubes into paths with prescribed endvertices. SIAM Journal of Discrete Mathematics, 22(4) (2008) 1448-1461.

[3] Egorychev, G. P. Proof of the van der Waerden conjecture for permanents. (Russian) Sibirsk. Mat. Zh. 22 (1981), no. 6, 6571, 225.

[4] Falikman, D. I. Proof of the van der Waerden conjecture on the permanent of a doubly stochastic matrix. (Russian) Mat. Zametki 29 (1981), no. 6, 931938, 957.

[5] Feder, Tomás and Subi, Carlos, Nearly Tight Bounds on the Number of Hamiltonian Circuits of the Hypercube and Generalizations. Electronic Colloquium on Computational Complexity, Report No. 87 (2008).

[6] J. Fink. Perfect matchings extend to Hamilton cycles in hypercubes. J. Comb. Theory, Ser. B, 97(6):1074-1076, 2007.

[7] J. Fink. Ph.D. Thesis, Charles University, Prague, Czech Republic. http://kam.mff.cuni.cz/ fink/publications/phdthesis.pdf. 
[8] D. G. Fon-Der-Flaass, Extending pairings to Hamiltonian cycles, Sib. Élektron. Mat. Izv., 7 (2010) 115-118.

[9] P. Gregor, Perfect matchings extending on subcubes to hamiltonian cycles of hypercubes, Discrete Math. 309 (2009) 1711-1713.

[10] P. Gregor and R. Škrekovski. On generalized middle level problem. Information Sciences, 180(12) (2010) 2448-2457.

[11] R. Häggkvist, On F-Hamiltonian graphs, in: J.A. Bondy and U.S.R. Murty, eds. Graph Theory and Related Topics (Academic Press, New York) (1979) 219-231.

[12] R. Häggkvist and C. Thomassen, Circuits through specified edges, Discrete Mathematics 41 (1982) $29-34$.

[13] B.Jackson and N. C. Wormald, Cycles containing matchings and pairwise compatible Euler tours, J. Graph Theory, 14(1) (1990) 127-138.

[14] G. Kreweras, Matchings and Hamiltonian cycles on hypercubes, Bull. Inst. Combin. Appl. 16 (1996), 87-91.

[15] S. C. Locke, R. Stong, Spanning Cycles in Hypercubes, 10892, The Amer. Math. Monthly 110 (2003) 440-441.

[16] F. Ruskey and C. D. Savage, Hamilton cycles that extend transposition matchings in Cayley graphs of $S_{n}$. SIAM Journal on Discrete Mathematics 6, No.1 (1993) 152-166. 